\title{
Spectral Properties of Schrödinger Operators with a Strongly Attractive $\delta$ Interaction Supported by a Surface
}

\author{
Pavel Exner
}

\begin{abstract}
We investigate the operator $-\Delta-\alpha \delta(x-\Gamma)$ in $L^{2}\left(\mathbb{R}^{3}\right)$, where $\Gamma$ is a smooth surface which is either compact or periodic and satisfies suitable regularity requirements. We find an asymptotic expansion for the lower part of the spectrum as $\alpha \rightarrow \infty$ which involves a "two-dimensional" comparison operator determined by the geometry of the surface $\Gamma$. In the compact case the asymptotics concerns negative eigenvalues, in the periodic case Floquet eigenvalues. We also give a bandwidth estimate in the case when a periodic $\Gamma$ decomposes into compact connected components. Finally, we comment on analogous systems of lower dimension and other aspects of the problem.
\end{abstract}

\section{Introduction}

The aim of the present paper is to discuss asymptotic spectral properties of a class of generalized Schrödinger operators in $L^{2}\left(\mathbb{R}^{3}\right)$. The corresponding potential will be a negative multiple of the Dirac measure supported by a surface $\Gamma \subset \mathbb{R}^{3}$. In other words, we are going to treat operators corresponding to the formal expression

$$
-\Delta-\alpha \delta(x-\Gamma),
$$

where $\alpha>0$ is independent of $x$; properties of $\Gamma$ will be specified below.

Apart from being an interesting mathematical question in itself, the problem has a natural motivation coming from quantum mechanics. Long time ago, physicists considered a formal "shrinking limit" for a particle localized in the vicinity of a manifold as a natural approach to quantization [JK, To, dC]. These considerations inspired studies of spectral and scattering properties of "fat" curved manifolds - see [DE, DEK] and references therein. Recently the mentioned limiting argument was reconsidered on a rigorous footing [FH], and related results were obtained for other geometric structures such as planar graphs $\mathbf{R u S}, \mathbf{K Z}$. The operator which played role of the Hamiltonian in these models was (a multiple of) the Dirichlet Laplacian in a neighborhood of the manifold. Sometimes other

1991 Mathematics Subject Classification. Primary 81Q05; Secondary 35J10, 35P15, 58J05.

Key words and phrases. Schrödinger operators, singular interactions, geometry.

The author obtained a support from NSF for a part of conference expenses and from GAAS Grant \#1048101. 
boundary conditions were used: in the papers $[\mathbf{R u S}, \mathbf{K Z}]$ the Dirichlet boundary condition was replaced by the Neumann one.

Another natural physical application, namely modeling of electron behavior in quantum wires, semiconductor thin films, and similar structures, motivates us to consider a modification of the above scheme in which the particle would be less strictly coupled to the manifold. A way to achieve this goal is to adopt the operator (1.1) as the Hamiltonian of such a system. It is clear that the confinement in this model takes place at negative energies only. Moreover, such a particle can be found at large distances from $\Gamma$, although with a small probability, because the exterior of the manifold is a classically forbidden region.

The "shrinking limit" then corresponds to making the $\delta$ coupling strong. The main idea is that for large $\alpha$ the eigenfunctions of the operator (1.1) are localized close to $\Gamma$. We employ a two-sided estimate of the eigenvalues using minimax principle in combination with a bracketing argument. We take a layer-type neighborhood of $\Gamma$ and impose Dirichlet and Neumann conditions at its boundary. In view of the strong localization we obtain in this way precise bounds for the negative part of the spectrum. Using the sketched method we have been able to get asymptotic behavior of the eigenvalues as $\alpha \rightarrow \infty$ for the operator (1.1) in $L^{2}\left(\mathbb{R}^{2}\right)$ with $\Gamma$ belonging to various curve classes - see EY1, EY2 and EY3 for planar loops with a magnetic field. In [EK1 the argument was extended to surfaces in $\mathbb{R}^{3}$ which are diffeomorphic to $\mathbb{R}^{2}$ and asymptotically planar; the aim was to show that under additional assumptions the operator (1.1) has then a nontrivial discrete spectrum ${ }^{1}$.

In the present paper we are going to treat the analogous problem for two other classes of manifolds without a boundary. The first are compact surfaces, the second periodic ones; we will derive an asymptotic expansion for the eigenvalues in the former case and for Floquet eigenvalues in the latter. An important question for periodic systems is the existence of spectral gaps. In case of a nontrivial periodic curve [EY2] open gaps always exist for $\alpha$ large enough. This is not true for surfaces. However, we will be able to prove the existence of gaps for a class of non-connected $\Gamma$. In conclusion we will comment on extensions of the mentioned two-dimensional results and some other aspects of the problem.

\section{Compact surfaces}

2.1. Formulation of the problem and the results. Let $\Gamma \subset \mathbb{R}^{3}$ be a $C^{4}$ smooth compact Riemann surface of a finite genus $g$, i.e. diffeomorphic to a sphere with $g$ handles attached [Kli. As such, it can be parameterized by a finite atlas. The $i$-th chart $p_{i}: U_{j} \rightarrow \mathbb{R}^{3}$ can be expressed in local coordinates $s_{\mu}^{(i)}, \mu=1,2$, the particular choice of which will not be important in the following. Usually, we will suppress the chart index. The metric tensor given in the local coordinates by $g_{\mu \nu}=p_{, \mu} \cdot p_{, \nu}$ defines the invariant surface area element $d \Gamma:=g^{1 / 2} d^{2} s$, where $g:=\operatorname{det}\left(g_{\mu \nu}\right)$. Furthermore, the tangent vectors $p_{, \mu}$ are linearly independent, and their cross product $p_{, 1} \times p_{, 2}$ gives, after rescaling, a unit normal field $n$ on $\Gamma$. The Weingarten tensor is then obtained by raising the index in the second fundamental form, $h_{\mu}{ }^{\nu}:=-n_{, \mu} \cdot p_{, \sigma} g^{\sigma \nu}$, where $\left(g^{\mu \nu}\right)$ means conventionally $\left(g_{\mu \nu}\right)^{-1}$. The eigenvalues $k_{ \pm}$of $\left(h_{\mu}{ }^{\nu}\right)$ are the principal curvatures. They determine the Gauss

\footnotetext{
${ }^{1}$ The analogous result in the two-dimensional case was proved under weaker assumptions in EI and for a curve in $\mathbb{R}^{3}$ in EK2 ; in both cases the $\delta$ coupling is not required to be strong.
} 
curvature $K$ and mean curvature $M$ by

$$
K=\operatorname{det}\left(h_{\mu}{ }^{\nu}\right)=k_{+} k_{-}, \quad M=\frac{1}{2} \operatorname{Tr}\left(h_{\mu}^{\nu}\right)=\frac{1}{2}\left(k_{+}+k_{-}\right) .
$$

The object of our interest is the generalized Schrödinger operator with an attractive measure-type potential. The latter is a multiple of the Dirac measure $\mu_{\Gamma}$ defined by $\mu_{\Gamma}(B):=\operatorname{vol}(B \cap \Gamma)$ for any Borel $B \in \mathbb{R}^{3}$, where $\operatorname{vol}(\cdot)$ is two-dimensional Hausdorff measure on $\Gamma$. Using the trace map $W^{2,1}\left(\mathbb{R}^{3}\right) \rightarrow L^{2}\left(\mathbb{R}^{3}, \mu_{\Gamma}\right) \cong L^{2}(\Gamma, d \Gamma)$ which is well defined in view of a standard Sobolev embedding, and abusing slightly the notation, we can define the quadratic form

$$
q_{\alpha}[\psi]=\|\nabla \psi\|_{L^{2}\left(\mathbb{R}^{3}\right)}^{2}-\alpha \int_{\mathbb{R}^{3}}|\psi(x)|^{2} d \mu_{\Gamma}(x), \quad \psi \in W^{2,1}\left(\mathbb{R}^{3}\right) .
$$

By Theorem 4.2 of $\mathbf{B E K S}$ this form is bounded from below and closed. Therefore, it is associated with a unique semibounded self-adjoint operator $H_{\alpha, \Gamma}$ which is regarded as the realization of the formal expression (1.1). Let us remark that since $\Gamma$ is smooth one can define the operator $H_{\alpha, \Gamma}$ alternatively through boundary conditions which involve the jump of the normal derivative across the surface in the same way as in EK1. This corresponds well to the physicist's concept of the $\delta$ interaction.

Since $\Gamma$ is compact by assumption, the essential spectrum of $H_{\alpha, \Gamma}$ equals $[0, \infty)$; our aim is to investigate the asymptotic behavior of the negative eigenvalues as $\alpha \rightarrow \infty$. It will be expressed in terms of the following comparison operator,

$$
S=-\Delta_{\Gamma}+K-M^{2}
$$

on $L^{2}(\Gamma, d \Gamma)$, where $\Delta_{\Gamma}=-g^{-1 / 2} \partial_{\mu} g^{1 / 2} g^{\mu \nu} \partial_{\nu}$ is the Laplace-Beltrami operator on $\Gamma$. We denote the $j$-th eigenvalue of $S$ as $\mu_{j}$. Notice that it is bounded from above by the $j$-th eigenvalue of $\Delta_{\Gamma}$ because the effective potential

$$
K-M^{2}=-\frac{1}{4}\left(k_{+}-k_{-}\right)^{2} \leq 0 ;
$$

the two coincide when $\Gamma$ is a sphere. Our first result then reads as follows.

TheOREM 2.1. (a) $\# \sigma_{\mathrm{d}}\left(H_{\alpha, \Gamma}\right) \geq j$ holds for a fixed integer $j$ if $\alpha$ is large enough. The $j$-th eigenvalue $\lambda_{j}(\alpha)$ of $H_{\alpha, \Gamma}$ has then an expansion of the form

$$
\lambda_{j}(\alpha)=-\frac{1}{4} \alpha^{2}+\mu_{j}+\mathcal{O}\left(\alpha^{-1} \ln \alpha\right) \quad \text { as } \quad \alpha \rightarrow \infty .
$$

(b) The counting function $\alpha \mapsto \# \sigma_{\mathrm{d}}\left(H_{\alpha, \Gamma}\right)$ behaves asymptotically as

$$
\# \sigma_{\mathrm{d}}\left(H_{\alpha, \Gamma}\right)=\frac{|\Gamma|}{16 \pi} \alpha^{2}+\mathcal{O}(\alpha) \quad \text { for } \quad \alpha \rightarrow \infty,
$$

where $|\Gamma|$ is the Riemann area of the surface $\Gamma$.

2.2. Proof of Theorem 2.1, First we construct a family of layer neighborhoods of $\Gamma$. Let $\{n(x): x \in \Gamma\}$ be a field of unit vectors normal to the manifold. Such a field exists globally because $\Gamma$ is orientable. Define a map $\mathcal{L}: \Gamma \times \mathbb{R} \rightarrow \mathbb{R}^{3}$ by $\mathcal{L}(x, u)=x+u n(x)$. Since $\Gamma$ is smooth by assumption, it is easy to see that there is an $a_{1}>0$ such that for each $a \in\left(0, a_{1}\right)$ the restriction

$$
\mathcal{L}_{a}(x, u)=x+\text { un }(x), \quad(x, u) \in \mathcal{N}_{a}:=\Gamma \times(-a, a),
$$

is a diffeomorphism of $\mathcal{N}_{a}$ onto its image $\Omega_{a}=\left\{x \in \mathbb{R}^{3}: \operatorname{dist}(x, \Gamma)<a\right\}$. 
We fix $a \in\left(0, a_{1}\right)$ and estimate (the negative spectrum of) $H_{\alpha, \Gamma}$ using operators acting in the layer $\Omega_{a}$. To this aim we define the quadratic forms $\eta_{\alpha, \Gamma}^{ \pm}[\cdot]$ with the domains $D\left(\eta_{\alpha, \Gamma}^{+}\right)=W_{0}^{2,1}\left(\Omega_{a}\right)$ and $D\left(\eta_{\alpha, \Gamma}^{-}\right)=W^{2,1}\left(\Omega_{a}\right)$, respectively, which associate with a vector $\psi$ the value

$$
\|\nabla \psi(x)\|_{L^{2}\left(\Omega_{a}\right)}^{2}-\alpha \int_{\mathbb{R}^{3}}|\psi(x)|^{2} d \mu_{\Gamma}(x) .
$$

Both the forms are closed and bounded from below; we call the self-adjoint operators in $L^{2}\left(\Omega_{a}\right)$ associated with them $H_{\alpha, \Gamma}^{ \pm}$. With this notation we can employ the Dirichlet-Neumann bracketing argument $\left[\mathbf{R S}\right.$ ] which yields the bounds ${ }^{2}$

$$
-\Delta_{\Sigma_{a}}^{N} \oplus H_{\alpha, \Gamma}^{-} \leq H_{\alpha, \Gamma} \leq-\Delta_{\Sigma_{a}}^{D} \oplus H_{\alpha, \Gamma}^{+}, \quad \Sigma_{a}:=\mathbb{R}^{3} \backslash \overline{\Omega_{a}} .
$$

In the estimation operators the sets $\Omega_{a}$ and $\Sigma_{a}$ are decoupled, so $\sigma\left(H_{\alpha, \Gamma}^{ \pm}\right)$is the union of the two spectra. As long as we are interested in the negative eigenvalues, we may take into account $H_{\alpha, \Gamma}^{ \pm}$only, because the "exterior" operators $\Delta_{\Sigma_{d}}^{D}$ and $\Delta_{\Sigma_{d}}^{N}$ are positive by definition.

In the next step we make use of the natural curvilinear coordinates in $\Omega_{a}$. More specifically, we transform $H_{\alpha, \Gamma}^{ \pm}$by means of the unitary operator

$$
\hat{U} \psi=\psi \circ \mathcal{L}_{a}: L^{2}\left(\Omega_{a}\right) \rightarrow L^{2}\left(\mathcal{N}_{a}, d \Omega\right) .
$$

The measure $d \Omega$ is associated to the pull-back to $\mathcal{N}_{a}$ of the Euclidean metric tensor in $\Omega_{a}$. We denote this pull-back metric tensor by $G_{i j}$; it has the form

$$
G_{i j}=\left(\begin{array}{cc}
\left(G_{\mu \nu}\right) & 0 \\
0 & 1
\end{array}\right), \quad G_{\mu \nu}=\left(\delta_{\mu}^{\sigma}-u h_{\mu}^{\sigma}\right)\left(\delta_{\sigma}^{\rho}-u h_{\sigma}{ }^{\rho}\right) g_{\rho \nu},
$$

which yields $d \Omega:=G^{1 / 2} d^{2} s d u$ in local coordinates with $G:=\operatorname{det}\left(G_{i j}\right)$ given by

$$
G=g\left[\left(1-u k_{+}\right)\left(1-u k_{-}\right)\right]^{2}=g\left(1-2 M u+K u^{2}\right)^{2} .
$$

Let $(\cdot, \cdot)_{G}$ denote the inner product in the space $L^{2}\left(\mathcal{N}_{a}, d \Omega\right)$. Then the operators $\hat{H}_{\alpha, \Gamma}^{ \pm}:=\hat{U} H_{\alpha, \Gamma}^{ \pm} \hat{U}^{-1}$ in $L^{2}\left(\mathcal{N}_{a}, d \Omega\right)$ are associated with the forms $\psi \mapsto \eta_{\alpha, \Gamma}^{ \pm}\left[\hat{U}^{-1} \psi\right]$,

$$
\eta_{\alpha, \Gamma}^{ \pm}\left[\hat{U}^{-1} \psi\right]=\left(\partial_{i} \psi, G^{i j} \partial_{j} \psi\right)_{G}-\alpha \int_{\Gamma}|\psi(s, 0)|^{2} d \Gamma,
$$

and they differ by their domains, $W_{0}^{2,1}\left(\mathcal{N}_{a}, d \Omega\right)$ and $W^{2,1}\left(\mathcal{N}_{a}, d \Omega\right)$ for the \pm sign, respectively. As above the expression $\psi(s, 0)$ in (2.8) can be given natural meaning using the trace mapping from $W_{0}^{2,1}\left(\mathcal{N}_{a}, d \Omega\right)$ or $W^{2,1}\left(\mathcal{N}_{a}, d \Omega\right)$ to $L^{2}(\Gamma, d \Gamma)$.

It is also useful to remove the factor $1-2 M u+K u^{2}$ from the weight $G^{1 / 2}$ in the inner product of $L^{2}\left(\mathcal{N}_{a}, d \Omega\right)$. This is achieved by means of another unitary transformation, namely

$$
U \psi=\left(1-2 M u+K u^{2}\right)^{1 / 2} \psi: L^{2}\left(\mathcal{N}_{a}, d \Omega\right) \rightarrow L^{2}\left(\mathcal{N}_{a}, d \Gamma d u\right) .
$$

We will denote the inner product in $L^{2}\left(\mathcal{N}_{a}, d \Gamma d u\right)$ by $(\cdot, \cdot)_{g}$. The operators $B_{\alpha, \Gamma}^{ \pm}:=$ $U \hat{H}_{\alpha, \Gamma}^{ \pm} U^{-1}$ acting in $L^{2}\left(\mathcal{N}_{a}, d \Gamma d u\right)$ are associated with the forms $b_{\alpha, \Gamma}^{ \pm}$given by

\footnotetext{
${ }^{2}$ This is the conventional way of expressing the argument. A purist might object against inequalities between operators having different domains. However, they make sense in combination with the quadratic form version of the minimax principle which is what we really need.
} 
$b_{\alpha, \Gamma}^{ \pm}[\psi]:=\eta_{\alpha, \Gamma}^{ \pm}\left[(U \hat{U})^{-1} \psi\right]$ which again differ by their domains. A straightforward computation, analogous to that performed in [DEK], yields

$$
\begin{aligned}
& b_{\alpha, \Gamma}^{+}[\psi]=\left(\partial_{\mu} \psi, G^{\mu \nu} \partial_{\nu} \psi\right)_{g}+\left(\psi,\left(V_{1}+V_{2}\right) \psi\right)_{g}+\left\|\partial_{u} \psi\right\|_{g}^{2}-\alpha \int_{\Gamma}|\psi(s, 0)|^{2} d \Gamma, \\
& b_{\alpha, \Gamma}^{-}[\psi]=b_{\alpha, \Gamma}^{+}[\psi]+\int_{\Gamma} M_{a}(s)|\psi(s, a)|^{2} d \Gamma-\int_{\Gamma} M_{-a}(s)|\psi(s,-a)|^{2} d \Gamma
\end{aligned}
$$

for $\psi$ from $W_{0}^{2,1}\left(\Omega_{a}, d \Gamma d u\right)$ and $W^{2,1}\left(\Omega_{a}, d \Gamma d u\right)$, respectively. The quantity $M_{u}:=$ $(M-K u)\left(1-2 M u+K u^{2}\right)^{-1}$ here is the mean curvature of the parallel surface characterized by a fixed value of $u$, and

$$
V_{1}:=g^{-1 / 2}\left(g^{1 / 2} G^{\mu \nu} J_{, \nu}\right)_{, \mu}+J_{, \mu} G^{\mu \nu} J_{, \nu}, \quad V_{2}:=\frac{K-M^{2}}{\left(1-2 M u+K u^{2}\right)^{2}}
$$

with $J:=\frac{1}{2} \ln \left(1-2 M u+K u^{2}\right)$ is the effective curvature-induced potential [DEK].

The operators $B_{\alpha, \Gamma}^{ \pm}$associated with the forms (2.10) are still not easy to handle because the surface and transverse variables are not decoupled. To get a rougher, but still sufficient, estimate we notice that $1-2 M u+K u^{2}$ can be squeezed between the numbers $C_{ \pm}(a):=\left(1 \pm a \varrho^{-1}\right)^{2}$, where $\varrho:=\max \left(\left\{\left\|k_{+}\right\|_{\infty},\left\|k_{-}\right\|_{\infty}\right\}\right)^{-1}$. Consequently, the matrix inequality $C_{-}(a) g_{\mu \nu} \leq G_{\mu \nu} \leq C_{+}(a) g_{\mu \nu}$ is valid. Moreover, the first component of the effective potential behaves as $\mathcal{O}(a)$ for $a \rightarrow 0$. Hence we have $\left|V_{1}\right| \leq v a$ for some $v>0$, while $V_{2}$ can be squeezed between the functions $C_{ \pm}^{-2}(a)\left(K-M^{2}\right)$, both uniformly in the surface variables. These observations motivate us to define the estimation operators in the following way,

$$
\tilde{B}_{\alpha, a}^{ \pm}:=S_{a}^{ \pm} \otimes I+I \otimes T_{\alpha, a}^{ \pm}
$$

with

$$
S_{a}^{ \pm}:=-C_{ \pm}(a) \Delta_{\Gamma}+C_{ \pm}^{-2}(a)\left(K-M^{2}\right) \pm v a
$$

in $L^{2}(\Gamma, d \Gamma) \otimes L^{2}(-a, a)$, where $T_{\alpha, a}^{ \pm}$are associated with the quadratic forms

$$
\begin{aligned}
& t_{\alpha, a}^{+}[\psi]:=\int_{-a}^{a}\left|\partial_{u} \psi\right|^{2} d u-\alpha|\psi(0)|^{2}, \\
& t_{\alpha, a}^{-}[\psi]:=\int_{-a}^{a}\left|\partial_{u} \psi\right|^{2} d u-\alpha|\psi(0)|^{2}-c_{a}\left(|\psi(a)|^{2}+|\psi(-a)|^{2}\right) .
\end{aligned}
$$

In these relations $\psi$ belongs to $W_{0}^{2,1}(-a, a)$ and $W^{2,1}(-a, a)$, respectively. In distinction to (2.10) the coefficient $c_{a}:=2\left(\|M\|_{\infty}+\|K\|_{\infty} a\right)$ in the boundary term of the second expression is independent of the surface variables $s$. The operators (2.12) provide us with the sought estimate in view of the obvious inequalities

$$
\pm B_{\alpha, \Gamma}^{ \pm} \leq \pm \tilde{B}_{\alpha, a}^{ \pm} .
$$

Since $\tilde{B}_{\alpha, a}^{ \pm}$have separated variables their spectra express through those of their constituent operators. To deal with the transverse part, we employ a simple estimate the proof of which can be found in EY1.

Lemma 2.2. There are positive numbers $c, c_{N}$ such that each one of the operators $T_{\alpha, a}^{ \pm}$has a single negative eigenvalue $\kappa_{\alpha, a}^{ \pm}$satisfying the inequalities

$$
-\frac{\alpha^{2}}{4}\left(1+c_{N} \mathrm{e}^{-\alpha a / 2}\right)<\kappa_{\alpha, a}^{-}<-\frac{\alpha^{2}}{4}<\kappa_{\alpha, a}^{+}<-\frac{\alpha^{2}}{4}\left(1-8 \mathrm{e}^{-\alpha a / 2}\right)
$$


when the attraction is strong enough, $\alpha>c \max \left\{a^{-1}, c_{a}\right\}$.

On the other hand, the surface part requires the following result.

Lemma 2.3. The $j$-th eigenvalues of the operators $S_{a}^{ \pm}$satisfy the asymptotic bounds $\left|\mu_{j, a}^{ \pm}-\mu_{j}\right| \leq m_{j}^{ \pm} a$ with some positive $m_{j}^{ \pm}$for all a small enough.

Proof. We assume $a<\varrho$ so $C_{-}(a)$ is positive. Using the definitions of $S_{a}^{ \pm}$ and $C_{ \pm}(a)$ we get easily the asymptotic bound

$$
\left\|S_{a}^{ \pm}-C_{ \pm}(a) S\right\| \leq\left(v+\left(\|K\|_{\infty}+\|M\|_{\infty}^{2}\right) \varrho^{-1}\right) a+\mathcal{O}\left(a^{2}\right):=m(a) .
$$

Combing this inequality with the minimax principle we find that $\left|\mu_{j, a}^{ \pm}-C_{ \pm}(a) \mu_{j}\right|$ does not exceed $m(a)$. Using once more the definition of $C_{ \pm}(a)$ we conclude that

$$
\left|\mu_{j, a}^{ \pm}-\mu_{j}\right| \leq m(a)+a\left|\left(2 \varrho^{-1}+\varrho^{-2} a\right) \mu_{j}\right|,
$$

which implies the sought result for small $a$.

Armed with these prerequisites we can now prove the asymptotic expansion for eigenvalues of $H_{\alpha, \Gamma}$. By minimax principle they are squeezed between the respective negative eigenvalues of $\tilde{B}_{\alpha, a}^{ \pm}$. Since each of the operators $T_{\alpha, a}^{ \pm}$has a single negative eigenvalue, the latter are of the form $\kappa_{\alpha, a}^{ \pm}+\mu_{j, a}^{ \pm}$provided $a$ is small and $\alpha$ is large enough. For definiteness we suppose that these eigenvalues are ordered in the same way as the $\mu_{j, a}^{ \pm}$'s are. Choosing

$$
a=a(\alpha):=6 \alpha^{-1} \ln \alpha
$$

and making use of the above two lemmata we find that

$$
\kappa_{\alpha, a}^{ \pm}+\mu_{j, a}^{ \pm}=-\frac{1}{4} \alpha^{2}+\mu_{j}+\mathcal{O}\left(\alpha^{-1} \ln \alpha\right)
$$

holds in this case as $a \rightarrow 0$. Since $\Gamma$ is compact the spectrum of $S$ is purely discrete accumulating at infinity only. Hence, to any positive integer $j$ there is an $\alpha_{j}$ such that $\kappa_{\alpha, a}^{+}+\mu_{j, a}^{+}<0$ holds for $\alpha>\alpha_{j}$. Consequently, $\tilde{B}_{\alpha, a}^{+}$has at least $j$ negative eigenvalues and the same is, of course, true for $H_{\alpha, \Gamma}$. Furthermore, since the upper and lower bound (2.16) differ by the error term only, we arrive at the claim (a).

Using minimax principle again we infer that there is a two-sided estimate

$$
\# \sigma_{\mathrm{d}}\left(S_{a}^{+}\right)=\# \sigma_{\mathrm{d}}\left(\tilde{B}_{\alpha, a}^{+}\right) \leq \# \sigma_{\mathrm{d}}\left(H_{\alpha, \Gamma}\right) \leq \# \sigma_{\mathrm{d}}\left(\tilde{B}_{\alpha, a}^{-}\right)=\# \sigma_{\mathrm{d}}\left(S_{a}^{-}\right) .
$$

Using (2.12) together with the definition of $C_{ \pm}(a)$ and the fact that the effective potential is bounded we find that $\# \sigma_{\mathrm{d}}\left(S_{a}^{ \pm}\right)=\# \sigma_{\mathrm{d}}(S)(1+\mathcal{O}(a))$. Similarly, the counting function for the operator (2.3) coincides with that of $-\Delta_{\Gamma}$, up to the same error. Thus it suffices to employ the well-known Weyl formula - see, e.g., Ch2 to get the claim (b) and to conclude thus the proof.

2.3. Remarks. The assumption that the surface $\Gamma$ is connected was made mostly for the sake of simplicity. The argument leading to the asymptotic formula (2.4) modifies easily to the case when $\Gamma$ is a finite disjoint union of $C^{4}$ smooth compact Riemann surfaces of finite genera. The situation is, of course, substantially more complicated if the number of compact connected components is infinite; in the next section we will discuss the particular case when such a $\Gamma$ is periodic.

Furthermore, we have supposed that $\Gamma$ is a manifold without a boundary. This was important in deriving the asymptotic expansion (2.4) because otherwise the eigenvalues of $\mu_{j}$ of the comparison operator would not be properly defined. On 
the other hand, the formula (2.5) remains valid even if $\Gamma$ has a nonempty and smooth boundary. It can be seen by an easy modification of the above argument. If we construct the neighborhood $\Omega_{a}$ in the described way, it will have the boundary consisting of two parts. One of them, $\partial \Omega_{a}^{(1)}$, contains as before points having normal distance $a$ from $\Gamma$. The additional part, $\partial \Omega_{a}^{(2)}$, is a subset of the normal surface to $\Gamma$ at $\partial \Gamma$. The form domains of the estimation operators will be again $W_{0}^{2,1}\left(\Omega_{a}\right)$ and $W^{2,1}\left(\Omega_{a}\right)$, respectively. Consequently, the operators $H_{\alpha, \Gamma}^{ \pm}$will satisfy Dirichlet and Neumann conditions at the whole boundary. In particular, they will satisfy these condition at the additional part $\partial \Omega_{a}^{(2)}$ of the boundary, and the same will be true for the boundary conditions which $S_{a}^{ \pm}$must satisfy at $\partial \Gamma$. The eigenvalues of the last named operators no longer differ by an $\mathcal{O}(a)$ term only. However, the Weyl asymptotics entering (2.17) is the same in both cases, because the difference in the number of surface eigenvalues is hidden in the error term.

It is also instructive to compare the formula 2.5] with the known estimates on the number of eigenvalues for generalized Schrödinger operator with measure-type potentials such as the modified Birman-Schwinger bound given in BEK Sec. 4]. Our result is valid in the asymptotic regime of strong coupling only but by its very nature it has the correct semiclassical behavior. On the other hand, the mentioned bound holds for any $\alpha>0$ but solvable examples, for instance with $\Gamma$ being a sphere AGS, BEKS , show that it may be rather crude for $\# \sigma_{\mathrm{d}}\left(H_{\alpha, \Gamma}\right)>1$.

\section{Periodic surfaces}

3.1. Floquet decomposition. Let $\mathcal{T} \equiv \mathcal{T}_{r}(b)$ be a discrete Abelian group of translations of $\mathbb{R}^{3}$ generated by an $r$-tuple $\left\{b_{i}\right\}$ of linearly independent vectors, where $r=1,2,3$. The starting point for the decomposition is a basic period cell $\mathcal{C}$ of $\mathbb{R}^{3}$, which is a simply connected set such that $\mathcal{C}_{n}:=\mathcal{C}+\sum_{i} n_{i} b_{i}$ is disjoint with $\mathcal{C}$ for any $n=\left\{n_{i}\right\} \in \mathbb{Z}^{r}$ different from zero and $\bigcup_{n \in \mathbb{Z}^{r}} \mathcal{C}_{n}=\mathbb{R}^{3}$. It is precompact if and only if $r=3$. The simplest choice of such a period cell is

$$
\mathcal{C}=\left\{\sum_{i=1}^{r} t_{i} b_{i}: 0 \leq t_{i}<1\right\} \times\left\{b_{i}\right\}^{\perp} .
$$

The main geometric object of this section will be a $C^{4}$ smooth Riemann surface $\Gamma \subset \mathbb{R}^{3}$, not necessarily connected, which is supposed to be periodic, i.e. such that $\mathcal{T}$ acts isometrically on $\Gamma$ and the quotient space $\Gamma / \mathcal{T}$ is compact. A basic period cell of $\Gamma$ is defined generally in terms of the group $\mathcal{T}$ and its fundamental domain Ch1. In general the decomposition of $\Gamma$ into period cells is independent of the above decomposition of the Euclidean space. However, for our purpose it is important that the two are consistent. Hence we choose the period cell of $\Gamma$ in the form $\Gamma_{\mathcal{C}}:=\Gamma \cap \mathcal{C}$. It is clear that $\partial \Gamma_{\mathcal{C}}=\Gamma \cap \partial \mathcal{C}$ is generally nonempty, in particular, if $\Gamma$ is connected. The boundary is piecewise smooth if $\partial \mathcal{C}$ has the same property.

We are interested again in the generalized Schrödinger operator $H_{\alpha, \Gamma}$ with a $\delta$ interaction supported now by the periodic surface. It is defined as above by means of the quadratic form (2.2); recall that Theorem 4.2 of [BEKS used there does not require the compactness of $\Gamma$. As usual in a periodic situation our main tool 
will be the Floquet analysis. We introduce the family of quadratic forms

$$
\begin{aligned}
q_{\alpha, \theta}[\psi] & =\|\nabla \psi\|_{L^{2}(\mathcal{C})}^{2}-\alpha \int_{\mathcal{C}}|\psi(x)|^{2} d \mu_{\Gamma}(x), \\
\operatorname{Dom}\left(q_{\alpha, \theta}\right) & =\left\{\psi \in W^{2,1}(\mathcal{C}): \psi\left(x+b_{i}\right)=\mathrm{e}^{i \theta_{i}} \psi(x)\right\},
\end{aligned}
$$

where $\theta=\left\{\theta_{i}\right\} \in[0,2 \pi)^{r}$, and denote by $H_{\alpha, \theta}$ the self-adjoint operators associated with them. For simplicity we will not indicate the dependence of these forms and operators on $\Gamma$. Modifying the standard reasoning [RS, EY2] to the present situation we get the sought decomposition.

Lemma 3.1. There is a unitary map $\mathcal{U}: L^{2}\left(\mathbb{R}^{3}\right) \rightarrow \int_{[0,2 \pi)^{r}}^{\oplus} L^{2}(\mathcal{C}) d \theta$ such that

$$
\mathcal{U} H_{\alpha, \Gamma} \mathcal{U}^{-1}=\int_{[0,2 \pi)^{r}}^{\oplus} H_{\alpha, \theta} d \theta
$$

and

$$
\sigma\left(H_{\alpha, \Gamma}\right)=\bigcup_{[0,2 \pi)^{r}} \sigma\left(H_{\alpha, \theta}\right) .
$$

The spectrum of $H_{\alpha, \theta}$ is purely discrete if $r=3$ while $\sigma_{\mathrm{ess}}\left(H_{\alpha, \theta}\right)=[0, \infty)$ if $r=1,2$; the eigenvalues (conventionally arranged in the ascending order, with their multiplicity taken into account) are continuous functions of the quasimomenta $\theta_{i}$.

Consequently, behavior of the spectral bands of $H_{\alpha, \Gamma}$ can be found through properties of eigenvalues of the fiber operators. The difference between the situation with $r=3$ and the "partially periodic" cases, $r=1,2$, is that in the former we will get the asymptotic behavior for all bands. Of course, the error term will not be uniform in the band index. Another difference is that in the case $r=3$ the spectrum is known to be absolutely continuous $\mathbf{S} \breve{\mathbf{S}}$, even under weaker assumptions than used here, while for $r=1,2$ this remains to be an open problem.

3.2. Fiber operator eigenvalues asymptotics. As before we need a comparison operator. In the present case it is defined on $L^{2}\left(\mathcal{C}, \mu_{\Gamma}\right) \cong L^{2}\left(\Gamma_{\mathcal{C}}, d \Gamma\right)$ by

$$
S_{\theta}=-\Delta_{\Gamma}+K-M^{2}
$$

with the domain consisting of those $\phi \in W^{2,1}\left(\Gamma_{\mathcal{C}}\right)$ with $\Delta_{\Gamma} \phi \in L^{2}\left(\Gamma_{\mathcal{C}}, d \Gamma\right)$. If $\Gamma_{\mathcal{C}}$ has a nontrivial boundary we have to require in addition that $\phi$ satisfies the Floquet conditions at the points of $\partial \Gamma_{\mathcal{C}}$. One can always choose the atlas in such a way that the local charts are periodic with respect to the group $\mathcal{T}$. In that case the conditions read $\phi\left(x+b_{i}\right)=\mathrm{e}^{i \theta_{i}} \phi(x)$ and

$$
\frac{\partial \phi\left(x+b_{i}\right)}{\partial s_{\mu}}=\mathrm{e}^{i \theta_{i}} \frac{\partial \phi(x)}{\partial s_{\mu}}, \quad 1 \leq i \leq r, \quad \mu=1,2,
$$

for derivatives with respect to the surface coordinates ${ }^{3}$. Since $\Gamma_{\mathcal{C}}$ is precompact and the curvatures involved are bounded, the spectrum of $S_{\theta}$ is purely discrete for each $\theta \in[0,2 \pi)^{r}$; we denote the $j$-th eigenvalue of $S_{\theta}$ as $\mu_{j}(\theta)$.

\footnotetext{
${ }^{3}$ One can also use a coordinate-free way, for instance, by bending the elementary cell into a torus and moving the quasimomentum from the boundary conditions into the operator.
} 
THEOREM 3.2. Under the stated assumptions the following claims are valid:

(a) Fix $\lambda$ as an arbitrary number if $r=3$ and a non-positive one for $r=1,2$. To any $j \in \mathbb{N}$ there is $\alpha_{j}>0$ such that $H_{\alpha, \theta}$ has at least $j$ eigenvalues below $\lambda$ for any $\alpha>\alpha_{j}$ and $\theta \in[0,2 \pi)^{r}$. The $j$-th eigenvalue $\lambda_{j}(\alpha, \theta)$ has then the expansion

$$
\lambda_{j}(\alpha, \theta)=-\frac{1}{4} \alpha^{2}+\mu_{j}(\theta)+\mathcal{O}\left(\alpha^{-1} \ln \alpha\right) \quad \text { as } \quad \alpha \rightarrow \infty,
$$

where the error term is uniform with respect to $\theta$.

(b) If the set $\sigma(S):=\bigcup_{\theta \in[0,2 \pi)^{r}} \sigma\left(S_{\theta}\right)$ has a gap separating a pair of bands, then the same is true for $\sigma\left(H_{\alpha, \Gamma}\right)$ if $\alpha$ is large enough.

Proof. The argument is the same as in Section 2.2 one has just to modify the domains of the quadratic forms involved and to check that the used estimates are uniform in $\theta$ which follows from the continuity of the Floquet eigenvalues.

3.3. Compactly disconnected periodic surfaces. By the second part of Theorem 3.2 the operator $H_{\alpha, \Gamma}$ has open spectral gaps in the asymptotic regime if the comparison operator has the same property. The latter may or may not be true depending on the geometry of $\Gamma$. The situation is different, however, if $\Gamma$ is not connected and each one of its connected component is compact and contained in (an interior of) a translate of the period cell $\mathcal{C}$. Let us stress that the last named property is a nontrivial assumption; to see that this is the case imagine a family of annular surfaces interlaced neighborwise into an infinite periodic "chain" 4 . An equally important observation is that while in Section 3.2 the choice of the basic period cell $\mathcal{C}$ was mostly irrelevant and one could settle for the simplest one represented by (3.1), it clearly matters here. To give an example ${ }^{5}$ consider an infinite array of a boomerang-shaped surfaces: they cannot be stacked in individual rectangular boxes if one wants them to be close enough to each other.

If the assumptions of this section are valid then the domain of the comparison operator is independent of the Floquet conditions (3.6). In that case, $S_{\theta}$ does not depend on $\theta$, and it has the form of a finite direct sum of operators of the type (2.3) for finite-genae surfaces to which the basic period cell $\Gamma_{\mathcal{C}}$, now automatically closed, can be decomposed (we have noted that in the first remark of Section 2.3). On the other hand, the Floquet decomposition (3.3) of the operator $H_{\alpha, \Gamma}$ is nontrivial and its spectral bands have generically nonzero widths. By the absolute continuity result mentioned above, we know that this is always true in the "fully periodic" case, $r=3$, while for $r=1,2$ the analogous claim is presently just a conjecture.

An easy way to estimate the spectral band widths is to employ a bracketing argument again. Inspecting the domains of the quadratic forms (3.2) we see that the Floquet eigenvalues can be bound from above and below if the boundary conditions for the fiber operator $H_{\alpha, \theta}$ are changed to Dirichlet and Neumann, respectively. Since $\operatorname{dist}\left(\partial \mathcal{C}, \Gamma_{\mathcal{C}}\right)>0$ holds by assumption, the neighborhood $\Omega_{a}$ of $\Gamma_{\mathcal{C}}$ is contained in the interior of $\mathcal{C}$ for all $a>0$ small enough. The negative part of the spectrum of the two estimation operators can be then treated in the exactly the same way as in the case of a compact surface, singly or finitely connected, because the "exterior"

\footnotetext{
${ }^{4}$ In fact the proof of Theorem 3.3 can be modified to this case too. We use this assumption to avoid a cumbersome formulation needed in more general situations.

${ }^{5}$ A proper name would be an "Australian gift shop" example.
} 
region $\Gamma_{\mathcal{C}} \backslash \mathcal{N}(a)$ contributes to the positive part only (more exactly, from the first eigenvalue up if $r=3)$. We arrive thus at the following result.

THEOREM 3.3. Let $\Gamma$ be a $C^{4}$ smooth periodic surface such that each one of its connected component is compact and contained in (an interior of) a translate of a fixed period cell $\mathcal{C}$ of the group $\mathcal{T}$. Denote by $\mu_{j}$ the $j$-th eigenvalue of the comparison operator (3.5). Then the $j$-th Floquet eigenvalue $\lambda_{j}(\alpha, \theta)$ from the decomposition (3.3) of the operator $H_{\alpha, \Gamma}$ behaves asymptotically as

$$
\lambda_{j}(\alpha, \theta)=-\frac{1}{4} \alpha^{2}+\mu_{j}+\mathcal{O}\left(\alpha^{-1} \ln \alpha\right) \quad \text { for } \quad \alpha \rightarrow \infty,
$$

with the error term uniform with respect to the quasimomenta $\theta$. Consequently, the number of open gaps in $\sigma\left(H_{\alpha, \Gamma}\right)$ exceeds any fixed integer if $\alpha$ is large enough.

\section{Concluding remarks}

4.1. Curves in the plane. A two-dimensional analogue of the present results was discussed in EY1, EY2, with $\Gamma$ being a smooth loop or an infinite smooth connected periodic curve. We have derived asymptotic expansions of the form (2.4) and (3.7) where $\mu_{j}$ and $\mu_{j}(\theta)$, respectively, are eigenvalues of the operator

$$
S=-\partial_{s}^{2}-\frac{1}{4} k(s)^{2} .
$$

Here $s$ is the arc length variable and $k$ is the signed curvature of $\Gamma$. The boundary conditions were periodic for the loop and the Floquet ones over the period in the other case. As in the three-dimensional situation, it is easy to extend these results to Hamiltonians with the $\delta$ interaction supported by a family of curves, be it a finite number of nonintersecting loops or a periodic system with multiple curves ${ }^{6}$.

The latter includes families of curves periodic in two directions, i.e. $r=2$ in the terminology of the preceding section. In that case we know from BSS that the spectrum of $H_{\alpha, \Gamma}$ is purely absolutely continuous so none of the spectral bands is degenerate; for $r=1$ this is an open problem again. From the viewpoint of open gaps, it is the absence of noncompact connected components of $\Gamma$ which is important. If $\Gamma$ can be broken into finite families of loops confined within the interior of the period cells, the analogue of Theorem 3.3 is valid and the system has many gaps for large $\alpha$. By [EY2 a single periodic connected curve which is not a straight line gives rise to an open gap for large $\alpha$, because the comparison operator (4.1) has the same property. It is not a priori clear whether the same is true for two or more such curves, because then we compare with a union of band spectra in which the gaps in one component may overlap with bands in the other one. It is not excluded, of course, that some gaps may survive since the curves have the same periodicity group; the problem deserves a deeper investigation.

4.2. Semiclassical interpretation. The results discussed here and in the earlier work mentioned in the introduction can be viewed also from a different perspective. Recall that the deviation of the spectrum of $H_{\alpha, \Gamma}$ from the one corresponding to the ideal manifold described by the comparison operator (2.3) are due to quantum tunneling. Hence they must be sensitive to the appropriate parameter,

\footnotetext{
${ }^{6} \mathrm{~A}$ model similar to the last named case was treated by a different technique in $\mathbf{K K}$ and earlier in FK. The setting used in these papers differs slightly from the present one. It concerns the roles of the coupling and spectral parameters which are switched there.
} 
i.e. the Planck's constant if we reintroduce it into the picture. However, the operator $-h^{2} \Delta-v \delta(x-\Gamma)$ is the $h^{2}$ multiple of (1.1) if we denote $\alpha:=v h^{-2}$. In this sense the obtained asymptotic formulae represent a semiclassical approximation.

4.3. Open problems. One can ask whether the "wide" gaps which one has if $\alpha$ is large and $\Gamma$ is decomposed into compact components will persist when the assumption about non-connected character of $\Gamma$ is weakened. It is natural to conjecture that the answer depends on properties of the corresponding operator (2.3). For the Laplace-Beltrami operator a construction of connected periodic manifolds exhibiting gaps has been presented recently Po1. It is based on connecting compact components of a non-connected surface by thin cylinders. It is worth examining what will be the effect of the curvature-induced potential $K-M^{2}$ which represents additional "potential wells" at the cylinders and connecting necks.

Spectral properties of the Laplace-Beltrami operator were studied also for other surface classes such as locally perturbed periodic ones for which eigenvalues in the gaps may appear Po2. A similar behavior may be expected for the operator (2.3) and one can ask whether the same will be true asymptotically for the corresponding $H_{\alpha, \Gamma}$. Our present method can yield information only on eigenvalues below the threshold of $\sigma_{\mathrm{ess}}\left(H_{\alpha, \Gamma}\right)$ because it employs the minimax principle in a substantial way, so another approach is needed.

An extension to higher dimensions, to an $m$-dimensional $\Gamma$ in $\mathbb{R}^{n}$, is also interesting. One conjectures that a similar asymptotic formula will be valid with the effective potential replaced by the function of the principal curvatures derived in [To, see also $[\mathbf{F H}]$. It is needed, however, that the operator corresponding to the symbol (1.1) makes sense. If the $\operatorname{codim} \Gamma=n-m$ equals one, it is defined as here in terms of quadratic forms. If $n-m=2,3$ one can proceed as in EK2 using generalized boundary conditions (and $-\alpha^{2} / 4$ will be replaced by the point-interaction eigenvalue in dimension $n-m$ ); for codim $\Gamma>3$ there is no meaningful operator $H_{\alpha, \Gamma}$, at least as long as we stay within the Hilbert-space theory.

Useful comments by S. Kondej, D. Krejčiř́ik, and P. Kuchment are gratefully acknowledged. I also appreciate the referee who checked every sentence twice at least.

\section{References}

[AGS] J.-P. Antoine, F. Gesztesy, J. Shabani: Exactly solvable models of spere interaction in quantum mechanics, J. Phys. A20 (1987), 3627-3712.

[BSŠ] M.S. Birman, T.A. Suslina, R.G. Shterenberg: Absolute continuity of the two-dimensional Schrödinger operator with delta potential concentrated on a periodic system of curves, Algebra i Analiz 12 (2000), 140-177; translated in St. Petersburg Math. J. 12 (2001), 535-567.

[BEKŠ] J.F. Brasche, P. Exner, Yu.A. Kuperin, P. Šeba: Schrödinger operators with singular interactions, J. Math. Anal. Appl. 184 (1994), 112-139.

[Ch1] I. Chavel, Riemannian Geometry; A Modern Perspective, Cambridge University Press 1993.

[Ch2] I. Chavel, The Laplacian on Riemannian manifolds, Proc. ICMS Instructional Conference "Spectral Theory and geometry", Cambridge University Press 1999, pp. 30-75.

[dC] R.C.T. da Costa: Quantum mechanics of a constrained particle, Phys. Rev. A23 (1981), $1982-1987$.

[DE] P. Duclos, P. Exner: Curvature-induced bound states in quantum waveguides in two and three dimensions, Rev. Math. Phys. 7 (1995), 73-102.

[DEK] P. Duclos, P. Exner, D. Krejčiřik: Bound states in curved quantum layers, Commun. Math. Phys. 223 (2001), 13-28.

[EI] P. Exner, T. Ichinose: Geometrically induced spectrum in curved leaky wires, J. Phys. A34 (2001), 1439-1450. 
[EK1] P. Exner, S. Kondej, Bound states due to a strong $\delta$ interaction supported by a curved surface, J. Phys. A36 (2003), 443-457.

[EK2] P. Exner, S. Kondej: Curvature-induced bound states for a $\delta$ interaction supported by a curve in $\mathbb{R}^{3}$, Ann. H. Poincaré 3 (2002), 967-981.

[EY1] P. Exner, K. Yoshitomi, Asymptotics of eigenvalues of the Schrödinger operator with a strong $\delta$-interaction on a loop, J. Geom. Phys. 41 (2002), 344-358.

[EY2] P. Exner, K. Yoshitomi, Band gap of the Schrödinger operator with a strong $\delta$-interaction on a periodic curve, Ann. H. Poincaré 2 (2001), 1139-1158.

[EY3] P. Exner, K. Yoshitomi, Persistent currents for 2D Schrödinger operator with a strong $\delta$-interaction on a loop, J. Phys. A35 (2002), 3479-3487.

[FK] A. Figotin, P. Kuchment: Band-gap structure of spectra of periodic dielectric and acoustic media I, II, SIAM J. Appl. Math. 56 (1996), 68-88, 1561-1620.

[FH] R. Froese, I. Herbst: Realizing holonomic constraints in classical and quantum mechanics, Commun. Math. Phys. 220 (2001), 489-535.

[JK] H. Jensen, H. Koppe: Quantum mechanics with constraints, Ann. Phys. 63 (1971), 586-591.

[Kli] W. Kligenberg, A Course in Differential Geometry, Springer Verlag, New York 1978.

[KK] P. Kuchment, L. Kunyansky: Spectral properties of high-contrast band-gap materials and operators on graphs, Experimental Math. 8 (1998), 1-28.

[KZ] P. Kuchment, Hongbiao Zeng: Convergence of spectra of mesoscopic systems collapsing onto a graph, J. Math. Anal. Appl. 258 (2001), 671-700.

[Po1] O. Post: Periodic manifolds with spectral gaps, J. Diff. Eq., to appear; math-ph/0207017.

[Po2] O. Post: Eigenvalues in spectral gaps of a perturbed periodic manifold, math-ph/0207018.

[RS] M. Reed, B. Simon, Methods of Modern Mathematical Physics, IV. Analysis of Operators, Academic Press, New York 1978.

[RuS] J. Rubinstein, M. Schatzman, Variational problems on multiply connected thin strips, I. Basic estimates and convergence of the Laplacian spectrum, Arch. Rat. Mech. Anal. 160 (2001), $271-308$.

[SŠ] T.A. Suslina, R.G. Shterenberg: Absolute continuity of the spectrum of the Schrödinger operator with the potential concentrated on a periodic system of hypersurfaces, Algebra i Analiz 13 (2001), 197-240.

[To] J. Tolar: On a quantum mechanical d'Alembert principle, in "Group Theoretical Methods in Physics", Lecture Notes in Physics, vol. 313, Springer, Berlin 1988; pp. 268-274.

Department of Theoretical Physics, Nuclear Physics Institute, Academy of Sciences, 25068 Řež near Prague, Czech Republic

E-mail address: exner@ujf.cas.cz 